\title{
EXCITATION ENERGY TRANSFER IN SYSTEMS OF MANY MOLECULES
}

\author{
Richard A. Cellarius \\ Biophysics Research Division, Institute of Science and Technology, University of Michigan, \\ Ann Arbor, Michigan
}

(Received 22 March 1966)

\begin{abstract}
Förster's theory of energy transfer in condensed systems is re-examined in the light of recent criticisms. It is concluded that his approach is correct and holds for a wide range of donor and quencher concentrations as long as there is no transfer among donor molecules. In contrast, the modified result of Tweet, Bellamy and Gaines, holds only for complete delocalization of the excitation among the donor molecules.
\end{abstract}

\section{INTRODUCTION}

THE resonance transfer of excitation energy theory of Förster ${ }^{(1)}$ states that the rate of transfer (probability of transfer per unit time) from an excited donor molecule, $D^{*}$, to an unexcited quencher (or acceptor) molecule, $Q$, is dependent on the inverse sixth power of the distance, $R$, between the two molecules:

$$
r_{t}=\frac{1}{\tau_{0}}\left(\frac{R_{0}}{R}\right)^{6}
$$

Here $\tau_{0}$ is the mean lifetime of $D^{*}$ in the absence of quenching and $R_{0}$ is the 'critical transfer distance', determined primarily by the overlap of the fluorescence spectrum of $D^{*}$ and the absorption spectrum of $Q$. A number of tests of this theory, using molecular 'sticks'(2) or thin films ${ }^{(3)}$ to hold the donor and quencher molecules a fixed distance apart, have been made, and these indicate the theory is essentially correct. Frequently, however, the donor and acceptor molecules are randomly distributed in a solution or in a two-dimensional film. In these cases, energy can be transferred from each excited donor to any one of a number of surrounding quencher molecules.

A number of methods have been used to estimate the total transfer or quenching in such systems. In some, the transfer only to nearest neighbor and next nearest neighbor molecules has been considered. ${ }^{(4)}$ Duysens, ${ }^{(5)}$ particularly, has discussed the case of transfer among the chlorophyll molecules in the chloroplast in these terms.

A more general approach has been taken by Förster, ${ }^{\left({ }^{8}\right)}$ by Galanin ${ }^{(7)}$ and by Rozman, ${ }^{\left({ }^{(8)}\right.}$ who average over all molecules in the system. Although each of these authors uses a different approach, their final expressions are identical, at least in the three-dimensional case of a solution. In a recent paper, however, Leibowitz ${ }^{(\theta)}$ discusses the correctness and incorrectness of the three approaches and concludes that Förster's approach is incorrect while Galanin's and Rozman's are correct. Since Förster's procedure was followed by Tweet, Bellamy and Gaines ${ }^{(10)}$ in estimating the transfer in two-dimensional monomolecular 
films of chlorophyll, it appeared important to determine whether the results of the three approaches differ in the two-dimensional case. In contradiction to Leibowitz's conclusion, I will show in section 3 that Förster's 'incorrect' and Rozman's 'correct' approaches are exactly equivalent for all distributions of molecules, either in two or three dimensions. Thus Förster's method, which is somewhat simpler, can be used to obtain the desired results. Galanin's method, as Leibowitz points out, contains an integral identical to Förster's and, in the approximations used, yields the same result in two or three dimensions. Galanin's method will not be examined further in this paper.

Another criticism of Förster's approach has been made by Tweet, Bellamy and Gaines. ${ }^{(10)}$ They claim his equation applies only when the donor concentration, $[D]$, is less than the quencher concentration, $[Q]$, and attempt to modify it for the case $[D]>[Q]$. Actually, Förster's result holds for the cases where $\left[D^{*}\right]<[Q]$ or $\left[Q^{*}\right]<[Q]$ and where there is no transfer among the donor molecules. The modified equation of Tweet, Bellamy and Gaines $(10)$ holds only for the limiting case where transfer among the donor molecules is so rapid that there is complete delocalization (exciton formation) among the donor molecules surrounding a given acceptor. This will be shown in section 4 .

\section{GENERAL}

We consider an excited donor molecule, $D_{1}^{*}$, surrounded by $N_{Q}$ quencher molecules in a finite volume, $V$. The rate of change of the probability, $\rho_{t}$, that $D_{i}$ is excited is

$$
\frac{\mathrm{d} \rho_{i}}{\mathrm{~d} t}=-\frac{1}{\tau_{e}} \rho_{i}-\frac{1}{\tau_{d}} \rho_{i}-\frac{1}{\tau_{0}} \rho_{i} \sum_{k=1}^{N_{0}}\left(\frac{R_{0 Q}}{R_{i k}}\right)^{6},
$$

where $1 / \tau_{e}$ is the rate constant for fluorescence and $1 / \tau_{d}$ is the rate constant for intersystem crossing and other non-radiative processes (excluding transfer). $\tau_{0}$ is the mean lifetime of

the excited molecule in the absence of quenching and $\frac{1}{\tau_{0}}=\frac{1}{\tau_{e}}+\frac{1}{\tau_{d}}$. Equation (2) is essentially that given by Forster. ${ }^{(\theta)}$ Integrating and taking $\rho_{t}(0)=1$, we obtain

$$
\rho_{i}(t)=\mathrm{e}^{-t / \tau_{0}} \exp \left[-\frac{t}{\tau_{0}} \sum_{k=1}^{N_{Q}}\left(\frac{R_{0} Q}{R_{i k}}\right)^{\sigma}\right] .
$$

The average probability,

$$
\overline{\rho(t)}=\frac{1}{n^{*}} \mathrm{e}^{-t / \tau_{0}} \sum_{i=1}^{n^{*}} \prod_{k=1}^{N_{Q}} \exp \left[-\frac{t}{\tau_{0}}\left(\frac{R_{0} Q}{R_{i k}}\right)^{0}\right],
$$

where $n^{*}$ is the number of donor molecules excited at $t=0$, is used to calculate the fluorescence yield:

$$
\eta(\mathcal{Q})=\frac{1}{\tau_{a}} \int_{0}^{\infty} \overline{\rho(t)} \mathrm{d} t
$$


The effect of energy transfer on the donor fluorescence is measured by comparing $\eta(Q)$ with $\eta_{0}$, the fluorescence yield in the absence of transfer:

$$
\eta_{0}=\frac{1}{\tau_{e}} \int_{0}^{\infty} \exp \left(-t / \tau_{0}\right) \mathrm{d} t=\tau_{0} / \tau_{e}
$$

and

$$
\frac{\eta(Q)}{\eta_{0}}=\frac{1}{\tau_{0}} \int_{0}^{\infty} \overline{\rho(t)} \mathrm{d} t
$$

3. THE CALCULATION OF $\bar{\rho}$ BY FORSTER AND BY ROZMAN

Instead of equation (4), Förster( ${ }^{(8)}$ writes

$$
\overline{\rho(t)}=\mathrm{e}^{-t / \tau_{0}} \prod_{k=1}^{N_{Q}} \int_{0}^{R_{g}} \exp \left[-\frac{t}{\tau_{0}}\left(\frac{R_{0 Q}}{R_{k}}\right)^{6}\right] \omega\left(R_{k}\right) \mathrm{d} R_{k}
$$

or

$$
\overline{\rho(t)}=\mathrm{e}^{-t / \tau_{a}}[J(t)]^{N_{Q}}
$$

with

$$
J(t)=\int_{0}^{R_{\theta}} \exp \left[-\frac{t}{\tau_{0}}\left(\frac{R_{0 Q}}{R}\right)^{6}\right] \omega(R) \mathrm{d} R .
$$

Here, $R_{g}$ is the radius of the sphere of volume $V$ containing the $N_{Q}$ quencher molecules and $\omega(R) \mathrm{d} R$ is the probability of finding a given quencher molecule in the region between $R$ and $R+\mathrm{d} R$ from the excited donor. This approach has been criticized ${ }^{(9)}$ on the grounds that instead of interchanging the sum (or integral) and product in equation (4), it is necessary to use the distribution function for the product $\prod_{k=1}^{N_{Q}} \exp \left[-\frac{t}{\tau_{0}}\left(\frac{R_{0 Q}}{R_{t k}}\right)^{6}\right]$ since different donors may have different distributions of quenchers around them. Denoting

$$
W_{i}^{Q}=\frac{t}{\tau_{0}} \sum_{k=1}^{N_{Q}}\left(\frac{R_{0 Q}}{R_{i k}}\right)^{6}
$$

and letting $\Phi(W) \mathrm{d} W$ be the probability that $W$ lies in the range between $W$ and $W+\mathrm{d} W$, with

$$
\int \Phi(W) \mathrm{d} W=1
$$

where the integration is taken over all accessible values of $W$, we obtain, from equation (4),

$$
\overline{\rho(t)}=\mathrm{e}^{-t / \tau_{0}} \int \mathrm{d} W^{Q} \Phi\left(W^{Q}\right) \exp \left(-t W^{Q}\right)
$$


This is the expression obtained by Rozman ${ }^{(8)}$, who evaluates $\Phi(W) \mathrm{d} W$ and $\rho$ by the Markov procedure outlined by Chandrasekar ${ }^{(11)}$. He obtains

$$
\Phi\left(W^{Q}\right) \mathrm{d} W^{Q}=\frac{\mathrm{d} W^{Q}}{2 \pi} \int_{-\infty}^{+\infty} \mathrm{d} x \exp \left(-i x W^{Q}\right) \int_{0}^{R_{q}} \ldots \int \prod_{k=1}^{N_{Q}}\left\{\exp \left[i x\left(R_{0 Q} / R_{k}\right)^{6}\right] \omega\left(R_{k}\right) \mathrm{d} R_{k}\right\} .
$$

In order to demonstrate the equivalence of Rozman's and Förster's procedures, instead of evaluating $\Phi\left(W^{Q}\right)$ further as does Rozman, we insert it directly into equation (12) and obtain, after some rearranging,

$$
\overline{\rho(t)}=\mathrm{e}^{-t / \tau_{0}} \int_{0}^{R_{0}} \ldots \int \prod_{k=1}^{N_{Q}}\left[\omega\left(R_{k}\right) \mathrm{d} R_{k}\right] \frac{1}{2 \pi} \int \mathrm{d} W \int_{-\infty}^{+\infty} \mathrm{d} x \exp (-t W) \exp \left\{i x\left[\sum_{k} \frac{1}{\tau_{0}}\left(\frac{R_{0 Q}}{R_{k}}\right)^{6}-W\right]\right\} .
$$

Using the Fourier integral theorem,

$$
f(y)=\frac{1}{2 \pi} \iint_{-\infty}^{+\infty} f(\zeta) \exp [i x(y-\zeta)] \mathrm{d} x \mathrm{~d} \zeta
$$

we obtain

$$
\overline{\rho(t)}=\mathrm{e}^{-t / \tau_{0}} \prod_{k=1}^{N_{Q}}\left\{\int_{0}^{R_{g}} \mathrm{~d} R_{k} \omega\left(R_{k}\right) \exp \left[-\frac{t}{\tau_{0}}\left(\frac{R_{0 Q}}{R_{k}}\right)^{6}\right]\right\},
$$

which is the same equation obtained by Förster [equation (8)]. It will be noted that in obtaining equation (8), by either method, we put no restrictions on $\omega(R)$. Thus Förster's procedure ${ }^{(8)}$ for calculating the quenching from equation $\left(8\right.$ or $\left.8^{\prime}\right)$ is generally applicable.

\section{THE RANGE OF APPLICABILITY OF FÖRSTER'S EQUATION}

Tweet, Bellamy and Gaines ${ }^{(10)}$ criticize Förster's final result on the basis that he "explicitly assume(s) that the quenching centers are more abundant than the fluorescent centers, $K_{c h l}<K_{Q}$ " where, in their notation, $K_{c h l}$ is the concentration of all the donors (not just the excited ones) and $K_{Q}$ is the quencher concentration. However, an unexcited donor molecule should not be considered to be a fluorescent center, and in most experiments (excluding perhaps flash photolysis and work with lasers) the concentration of excited donors is a small fraction of the total donor concentration, even when considered over a time interval several times $\tau_{0}$. Moreover, it does not appear necessary to restrict the volume $V$ (see section 2) to non-overlapping spheres of a diameter equal to the distance between two excited donor molecules. As long as the number of excited quenchers is small compared to the total number of quenchers, so that the quencher concentration, $[Q]$, and the distribution function, $\omega(R)$ are independent of light intensity and time, $V$ can be taken as the volume of the entire solution or at least some useful macroscopic fraction thereof. It is these restrictions on $\omega(R)$ and $[Q]$ which limit the range of applicability of Förster's final expressions. 
It is useful to examine the modified result of Tweet, Bellamy and Gaines ${ }^{(10)}$ more closely and determine its range of applicability. In contrast to our approach in section 2, they consider the case of a single quencher surrounded by a random distribution of $N_{D}$ donors in the volume $V_{D}$. In this case, from equation (2), we would obtain, with only one quencher near an excited donor,

$$
\frac{\mathrm{d} \rho t}{\mathrm{~d} t}=-\frac{\rho_{t}}{\tau_{0}}-\frac{\rho_{i}}{\tau_{0}}\left(\frac{R_{0} Q}{R_{i}}\right)^{6}
$$

assuming no interaction among the donors.

However, Tweet, Bellamy and Gaines write that the rate of change of the probability that a given donor molecule is excited is

$$
\frac{\mathrm{d} \rho}{\mathrm{d} t}=-\frac{\rho}{\tau_{0}}-\frac{\rho}{\tau_{0}} \sum_{i=1}^{N D} \frac{1}{N_{D}}\left(\frac{R_{0} Q}{R_{i}}\right)^{6}
$$

where they have "assumed that all donors have an equal probability of being excited." equation (16) indicates that the probability of transfer from a given donor is affected by neighboring donors or that the transfer of excitation among the donor molecules is so rapid as to preclude its localization on any particular donor. Thus all donors have the same probability of being excited, not only at the instant of light absorption, but at all times $t \geq 0$. This is equivalent to complete delocalization of the energy over the $N_{D}$ donor molecules. Thus the range of concentrations in which the result of Tweet, Bellamy and Gaines is applicable is restricted by this delocalization requirement. [In integrating equation (16) Tweet, Bellamy and Gaines assume $\rho(0)=1$. While there might be some question regarding the correctness of this assumption, it can easily be shown that as long as $p(0)$ is a constant, the final result will be no different from what they obtain. Similarly, by proceeding as in section 3 , one can show that they do obtain the correct results, even though following Förster's method.]

Acknowledgements-This investigation was supported by a Public Health Service Fellowship (Number 1-F2-GM-28,234-01) from the National Institute of General Medical Sciences. A portion of this work was done at the Department of Biophysics, University of Chicago. I am grateful to Professor John R. Platt and Dr. Jehuda Feitelson for comments and to Dr. Feitelson for translating the paper by Rozman. ${ }^{\left({ }^{(}\right)}$

\section{REFERENCES}

1. TH. FörSTER, Fluoreszenz Organischer Verbindungen. Vandenhoeck and Ruprecht, Göttingen (1951).

2. S. A. Latt, H. T. Cheung and E. R. Blout, J. Am. Chem. Soc. 87, 995 (1965).

3. K. H. Drexhage, M. M. Zwick and H. KuHN, Ber. Bunsenges. Phys. Chem. 67, 62 (1963).

4. A. ORE, J. Chem. Phys. 33, 31 (1960).

5. L. N. M. DuYsens, Progr. Biophys. 14, 1 (1964).

6. TH. Förster, $Z$. Naturforsch. 4a, 321 (1949).

7. M. D. GalANIN, Zhur. Eksptl. i Teoret. Fiz. 28, 485 (1955).

8. I. M. Rozman, Optika i Spektroskopiya 4, 536 (1958).

9. M. Lemowtiz, J. Phys. Chem. 69, 1061 (1965).

10. A. G. Tweet, W. D. Bellamy and G. L. Gaines, JR., J. Chem. Phys. 41, 2068 (1964).

11. S. Chandrasekhar, Rev. Mod. Phys. 15, 1 (1943). 